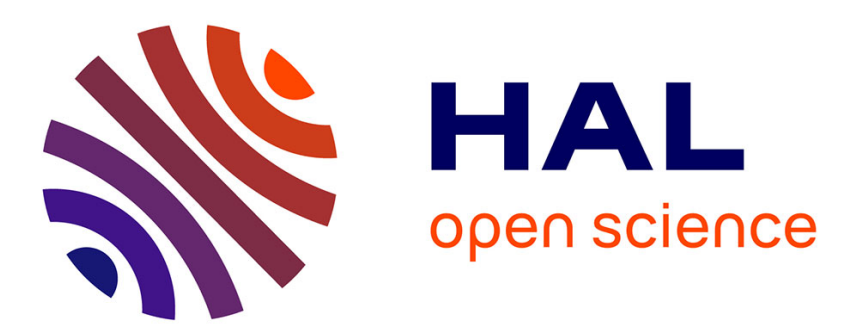

\title{
The masses of the first family of fermions and of the Higgs boson are equal to integer powers of 2
}

Nathalie Olivi-Tran

\section{To cite this version:}

Nathalie Olivi-Tran. The masses of the first family of fermions and of the Higgs boson are equal to integer powers of 2. Advanced Studies in Theoretical Physics, 2014, 8 (11), pp.511-516. 10.12988/astp.2014.4449. hal-00998959

\section{HAL Id: hal-00998959 \\ https://hal.science/hal-00998959}

Submitted on 3 Jun 2014

HAL is a multi-disciplinary open access archive for the deposit and dissemination of scientific research documents, whether they are published or not. The documents may come from teaching and research institutions in France or abroad, or from public or private research centers.
L'archive ouverte pluridisciplinaire HAL, est destinée au dépôt et à la diffusion de documents scientifiques de niveau recherche, publiés ou non, émanant des établissements d'enseignement et de recherche français ou étrangers, des laboratoires publics ou privés. 


\title{
The masses of the first family of fermions and of the Higgs boson are equal to integer powers of 2
}

\author{
Nathalie Olivi-Tran \\ Laboratoire Charles Coulomb, CNRS, \\ UMR 5221, place Eugene Bataillon, \\ 34095 Montpellier cedex 5, France, \\ Laboratoire Charles Coulomb, Universite Montpellier 2, \\ place Eugene Bataillon, 34095 Montpellier cedex 5, France \\ email: Nathalie.Olivi-Tran@univ-montp2.fr
}

(Dated: May 13, 2014)

\begin{abstract}
We noticed that the first family of fermions and the Higgs boson have masses which are equal to integer powers of 2 in $e V / c^{2}$ units (i.e. in the Planck length units). We made the hypothesis that, if spacetime is composed of small hypercubes of one Planck length edge, it exists elementary wavefunctions which are equal to $\sqrt{2} \exp \left(i k x_{i}\right)$ if it corresponds to a space dimension or equal to $\sqrt{2} \exp (i \omega t)$ if it corresponds to a time dimension. By using the Dirac propagation equation and combinatorics we showed that the electron has a mass of $2^{19} \mathrm{eV} / \mathrm{c}^{2}$, the quark has a mass of $2^{21} \mathrm{eV} / \mathrm{c}^{2}$ and the electron neutrino has a mass of $2 \mathrm{eV} / \mathrm{c}^{2}$. Finally, the Higgs boson is showed to have a mass of $2^{37} \mathrm{eV} / \mathrm{c}^{2}$
\end{abstract}

Keywords: theoretical masses of elementary particles;fourdimensional space; real space theory 


\section{INTRODUCTION}

Let us make the hypothesis that spacetime is fourdimensional and that time is equivalent to a space dimension (except that it is not possible to go backwards in time) [2-4]. We made a classification of elementary particles with respect to their dimensions [5]. Following the conclusions of this article, we analyze here the masses of some of these particles.

One may remark that the masses of the first family of fermions (elementary particles) and of the Higgs boson are integer powers of 2 if expressed in $e V / c^{2}$. For details on these masses, see table I.

\begin{tabular}{|c|c|c|}
\hline Name & measured mass in $e V / c^{2}$ & $2^{n}$ \\
\hline Electron & $\approx 511.10^{3}$ & $2^{19} \approx 524.10^{3}$ \\
Quark up & $\approx 2.3 .10^{6}$ & $2^{21} \approx 2.1 .10^{6}$ \\
Electron neutrino & $\approx 2$ & $2^{1}$ \\
Higgs boson & $\approx 126.10^{9}$ & $2^{37} \approx 137.10^{9}$ \\
\hline
\end{tabular}

TABLE I. Masses of the first family of fermions and of the Higgs boson [1]

In order to explain this property, let us analyze the Dirac equation which is the propagation equation of part of the first family of fermions (except the electron neutrino).

The Dirac equation may be written:

$$
i \hbar \gamma^{\mu} \partial_{\mu} \psi-m c \psi=0
$$

with $\psi$ the wavefunction, $m$ the mass of the fermion, $c$ the speed of light and with the Dirac matrices:

$$
\gamma^{0}=\left(\begin{array}{cc}
I_{2} & 0 \\
0 & -I_{2}
\end{array}\right), \gamma^{1}=\left(\begin{array}{cc}
0 & \sigma_{x} \\
-\sigma_{x} & 0
\end{array}\right), \gamma^{2}=\left(\begin{array}{cc}
0 & \sigma_{y} \\
-\sigma_{y} & 0
\end{array}\right), \gamma^{3}=\left(\begin{array}{cc}
0 & \sigma_{z} \\
-\sigma_{z} & 0
\end{array}\right)
$$

where $\sigma_{\nu}$ are the Pauli matrices.

On a mathematical point of view, the Dirac matrices are representative of infinitesimal rotations within the wavefunction of a given elementary particle.

Let us make two hypotheses: first, the elementary wave function corresponds to the eigenfunction of a square potential with dimensions corresponding to the Planck length (we take $\hbar=1$ here). This first hypothesis leads to elementary eigenfunctions which are equal to 
$\sqrt{2} \exp \left(i k x_{i}\right)$ if the eigenfunction corresponds to a space dimension or equal to $\sqrt{2} \exp (i \omega t)$ if the eigenfunction corresponds to a temporal dimension.

The second hypothesis may be found in a previous article by Olivi-Tran and Gottiniaux [5]. This second hypothesis depends on which fermion one deals with.

\section{MASS OF THE ELECTRON}

Following Olivi-Tran and Gottiniaux [5], the electron is fourdimensional. So, the wavefunction has four subcomponents which may be seen also as wavefunctions. In order to get the mass of the electron, let us analyze the Dirac equation (equation (1)) which may be rewritten:

$$
i \gamma^{\mu} \partial_{\mu} \psi=m c \psi
$$

The norm of the Dirac matrices is equal to one, so the mass $m$ of the electron is equal to the norm of the eigenfunctions of equation (3).A hint is to write equation (3) with the use of one unique matrix containing all Dirac matrices. Moreover, one has to take into account the fact that this large matrix has to contain all possible combinations of Pauli matrices . Thus there are 3 possibilities of arranging $\gamma^{1}, \gamma^{2}, \gamma^{3}$, and $6=3$ ! possibilities to permute the Pauli matrices within the Dirac matrices; all space dimensions are thus equivalent. In the case of $\sigma_{0}$ which is the temporal Pauli matrix, there is one possibility to arrange it.

So the large matrix containing all combinations of $\gamma^{\mu}$ has a dimension of $(3.6+1) .2=38$ :i.e. $38 X 38$ (we multiply $(3.6+1)$ by 2 because the Pauli matrices are twodimensional). If we recall that the subeigenfunctions (see section Introduction) have a norm equal to $\sqrt{2}$, the whole eigenfunction of equation (3) has a norm equal to $\sqrt{2}^{38}=2^{19} \mathrm{eV} / \mathrm{c}^{2}$. Indeed, the whole eigenfunction is the product of 38 subeigenfunctions, each of norm $\sqrt{2}$.

Finally, if one deals with $\mathrm{eV} / \mathrm{c}^{2}$ units, the mass of the electron is equal to $2^{19} \mathrm{eV} / \mathrm{c}^{2}$.

\section{MASS OF THE QUARK UP}

Following Olivi-Tran and Gottiniaux [5], the quark up is threedimensional. So, the wavefunction has three subcomponents which may be seen also as wavefunctions. In order to get the mass of the quark up, let us analyze the Dirac equation (equation (1)) which may be 
rewritten:

$$
i \gamma^{\mu} \partial_{\mu} \psi=m c \psi
$$

The norm of the Dirac matrices is equal to one, so the mass $m$ of the quark up is equal to the norm of the eigenfunctions of equation (4).A hint is to write equation (4) with the use of one unique matrix containing all Dirac matrices. Moreover, one has to take into account the fact that this large matrix has to contain all possible permutations of Pauli matrices. Thus there are $6=3$ ! possibilities to combine the Pauli matrices within the Dirac matrices, all space dimensions are thus equivalent. In the case of $\sigma_{0}$ which is the temporal Pauli matrix, there is one possibility to arrange it.

If all space dimensions are equivalent this leads to all possible combinations of $\gamma^{\mu}, \gamma^{\nu}, \gamma^{0}$. As the quark up is threedimensional (two spatial dimensions and one temporal dimension), one has to multiply the number of combinations by three (one for each couple of dimensions, i.e. $x, y$ and $y, z$ and $x, z$ ). So the large matrix containing all combinations has a dimension of $((6+1) \cdot 3) \cdot 2=42$ (we multiply $(6+1) .3$ by two because the Pauli matrices are twodimensional). If we recall that the subeigenfunctions (see section Introduction) have a norm equal to $\sqrt{2}$, the whole eigenfunction of equation (3) has a norm equal to $\sqrt{2}^{42}=2^{21} \mathrm{eV} / \mathrm{c}^{2}$. Indeed, the whole eigenfunction is the product of 42 subeigenfunctions, each of norm $\sqrt{2}$.

Finally, if one deals with $\mathrm{eV} / \mathrm{c}^{2}$ units, the mass of the quark up is equal to $2^{21} \mathrm{eV} / \mathrm{c}^{2}$.

\section{MASS OF THE ELECTRON NEUTRINO}

Following Olivi-Tran [5], the electron neutrino is twodimensional: one temporal dimension and one spatial dimension. Up to now, there is no existing theoretical propagation equation for the neutrinos. Let us make the hypothesis that all space dimensions are equivalent: as the electron neutrino has only one spatial dimension, there is no 'rotations' between different spatial dimensions (as for the electron and the quark). In the propagation equation for neutrino, there would be no Dirac matrices and only two subeigenfunctions would come into account: one for space the other for time. The number of subeigenfunctions (see previous sections) is then equal to two. A theoretical propagation function for the neutrino would then use 2 subfunctions $\sqrt{2} \exp (i \omega t)$ and $\sqrt{2} \exp (i k x)$. So the mass of the electron neutrino would be $\sqrt{2}^{2}=2 e \mathrm{~V} / \mathrm{c}^{2}$. 


\section{MASS OF THE HIGGS BOSON}

In a previous paper, Olivi-Tran [6] showed that the Higgs field corresponds to the solution of the massless Klein-Gordon propagation equation coupled to a hypercubic fourdimensional potential. The first family of fermions acquire mass by interacting with the Higgs field. So, the lowest mass which is possible for the Higgs boson corresponds to the lowest possible dimensions of the hypercubic Higgs field. The lowest spatial volume -smallest common multiple-(threedimensional) for the electron is $2^{18}$ (see section II), the lowest spatial volume -smallest common multiple-(twodimensional) for the quark is also $2^{18}$ (see section III), the lowest spatial dimension for electron neutrino is included in the spatial dimensions of the electron (see section IV). Finally, the lowest temporal dimension -smallest common multiplefor either the electron, the quark (for the electron neutrino this temporal dimension is included in the previous ones) is 2 . If we make the hypothesis that the hypervolume of the Higgs field corresponds to its mass, the mass of the Higgs boson would be equal to $2^{18} \cdot 2^{18} \cdot 2=2^{37} \mathrm{eV} / \mathrm{c}^{2}$

\section{CONCLUSION}

The masses of the first family of fermions and of the Higgs boson are integer powers of 2 within experimental errors. As what I wrote in [5], other fermions are excited states of the first family and thus they do not follow the same rule regarding their masses. Now, one has to analyze the masses of the $W$ and $Z$ bosons which maybe can be found theoretically with the same reasonings.

[1] Wikipedia -Elementary particles-April 2014

[2] N.Olivi-Tran and P.M.Gauthier, The FLRW cosmological model revisited: Relation on the local time with the local curvature and consequences on the Heisenberg uncertainty principle Adv. Studies Theor. Phys. vol.2 no 6 (2008) 267-270

[3] N.Olivi-Tran What if our three dimensional curved universe was embedded in four dimensional space? Consequences on the EPR paradox Adv. Studies Theor. Phys., Vol. 3, (2009), no. 12, $489-492$ 
[4] N.Olivi-Tran Dimensional analysis of Einstein's fields equations Adv. Studies Theor. Phys., Vol. 3, (2009), no. 1, 9 - 12

[5] N.Olivi-Tran and N.Gottiniaux A classification of elementary particles in $d=4$ following a simple geometrical hypothesis in real space Adv. Studies Theor. Phys., Vol. 7, 2013, no. 18, $853-857$

[6] N.Olivi-Tran Is it the Higgs scalar field? Advanced Studies in Theoretical Physics Vol. 4, 2010, no. $13,633-636$ 